1

2

3

4

5

6

7

8

9

10

11

12

13

14

15

16

17

18

19

20

21

22

23

24

25

26

27

28

29

30

31

32

33

34

35

36

37

38

39

40

41

42

43

44

45

46

47

48

49

50

51

52

53

54

55

56

\title{
Analysis of reaction intermediates in tryptophan 2,3-dioxygenase: a comparison with
} indoleamine 2,3-dioxygenase

\author{
Jaswir Basran ${ }^{{ }^{*}}$, Elizabeth S Booth ${ }^{\S *}$, Michael Lee ${ }^{\ddagger}$, Sandeep Handa ${ }^{\S}$, and Emma L. Raven ${ }^{1, \S}$ \\ * Department of Molecular and Cellular Biology and Henry Wellcome Laboratories for Structural \\ Biology, Henry Wellcome Building, University of Leicester, Lancaster Road, Leicester, LE1 7RH, \\ England UK. ${ }^{\S}$ Department of Chemistry, University of Leicester, University Road, Leicester, LE1 \\ 7RH, England UK. \\ ${ }^{*}$ These authors contributed equally to this work
}

\begin{abstract}
ABBREVIATIONS
IDO, indoleamine 2,3-dioxygenase; TDO, tryptophan 2,3-dioxygenase; L-Trp, L-tryptophan; NFK, Nformylkynurenine.
\end{abstract}




\section{ABSTRACT}

Indoleamine 2,3-dioxygenase (IDO) and tryptophan 2,3-dioxygenase (TDO) are heme-containing enzymes that catalyse the $\mathrm{O}_{2}$-dependent oxidation of L-tryptophan (L-Trp) in biological systems. Although many decades have passed since their discovery, the mechanism of tryptophan oxidation is not established. It has been widely assumed that IDO and TDO react using the same mechanism, although there is no evidence that they do. For IDO, a Compound II (ferryl) species accumulates in the steady state and is implicated in the mechanism; in TDO, no such species has ever been observed. In this paper, we examine the kinetics of tryptophan oxidation in TDO. We find no evidence for accumulation of Compound II during TDO catalysis. Instead, a ternary [Fe(II)- $\mathrm{O}_{2}$, L-Trp] complex is detected under steady state conditions. The absence of a Compound II species in the steady state in TDO is not due to an intrinsic inability of the TDO enzyme to form ferryl heme, because Compound II can be formed directly through a different route in which ferrous heme is reacted with peroxide. We interpret the data to mean that the rate-limiting step in the IDO and TDO mechanisms is not the same. 
In a seminal paper in $1957,{ }^{l}$ Hayaishi demonstrated that $\mathrm{O}_{2}$-dependent oxidation of tryptophan led to formation of $\mathrm{N}$-formylkynurenine (NFK), and that during this process two atoms of oxygen from $\mathrm{O}_{2}$ were incorporated into the substrate. The class of enzymes catalyzing this oxidative chemistry divide into two categories. The first - the indoleamine 2,3-dioxygenases (IDO) - have wide substrate specificity and are able to oxidise L-tryptophan (L-Trp) and a number of other closely related indolecontaining substrates. The second - the tryptophan 2,3-dioxygenases (TDO) - are much more particular in their choice of substrate, and typically oxidise just L-Trp ${ }^{2,3}$.

More than fifty years after Hayaishi's paper, the mechanism of tryptophan oxidation is not established. Early proposals ${ }^{4}$ suggested a base-catalysed abstraction mechanism and no change in oxidation state of the metal, with an active site histidine implicated. But several groups had independently reported ${ }^{5-7}$ that the 1-Me-L-Trp analogue, Scheme 1 , is also reactive, and it was noted ${ }^{5}$ that this is not consistent with a base-catalysed abstraction mechanism. Mutational data where the presumed active site histidine is missing were also not consistent with base-catalysed abstraction ${ }^{8}$.

For IDO, there is now good evidence that a ferryl (Compound II) species is formed during turnover. This Compound II species was first identified by resonance Raman during oxidation of L-Trp ${ }^{9,10}$, and more recently the uv-visible spectra have been extracted from kinetic analyses with a number of different substrates ${ }^{11}$. Computational analyses are also consistent with ferryl heme formation ${ }^{12-15}$.

It has been widely presumed that IDO and TDO oxidise substrates using the same mechanism, even though there is no evidence to support that assumption. There are, in fact, some substantive anomalies. Unlike IDO, a stable ferrous oxy-intermediate of TDO has not been identified. More significant is the fact that a Compound II intermediate has never been observed for any TDO. Hence, it remains to be established that the two enzymes use the same mechanism.

In this work, we have examined the transient intermediates formed on reaction of human TDO (hTDO) and $X$. campestris TDO (XcTDO) with substrate. Unlike IDO, we find no evidence for accumulation of a Compound II intermediate under turnover conditions in TDO; instead a ternary $\left[\mathrm{Fe}(\mathrm{II})-\mathrm{O}_{2}, \mathrm{~L}-\mathrm{Trp}\right]$ complex accumulates during turnover. We conclude that the rate-limiting step in the TDO mechanism is different from that in IDO. We discuss this information in the light of a broader framework for development of ideas on the mechanism of tryptophan oxidation in both IDO and TDO. 


\section{EXPERIMENTAL PROCEDURES}

Materials. All chemicals of analytical and reagent grade were obtained from commercial sources and used without further purification unless otherwise stated. All media, buffers and solutions were prepared using ultra-pure, doubly deionised water from an Elga PureLab water purifier.

Recombinant expression of TDO. The cloning of genes for production of human TDO (hTDO) and $X$. campestris TDO (XcTDO) has been reported previously ${ }^{16-18}$. Recombinant proteins were expressed in E. coli BL21 (DE3) cells. A $200 \mathrm{ml}$ starter culture of 2xYT-media supplemented with the appropriate antibiotic(s) was inoculated with a single colony from an LB-agar plate and incubated overnight at $37{ }^{\circ} \mathrm{C}$ with shaking at $200 \mathrm{rpm}$. The starter culture was used to inoculate $12 \mathrm{x} 600 \mathrm{ml}$ of 2xYT-media supplemented with the appropriate antibiotic(s). This larger culture was incubated at 37 ${ }^{\circ} \mathrm{C}$ with shaking at $200 \mathrm{rpm}$ until the absorbance at $600 \mathrm{~nm}\left(\mathrm{OD}_{600}\right)$ reached $\sim 0.8$. Protein expression was induced by the addition of IPTG $(0.2 \mathrm{mM})$. The cells were then incubated for 24 hours with shaking at $200 \mathrm{rpm}$ and the temperature reduced (to $25^{\circ} \mathrm{C}$ for hTDO and to $18{ }^{\circ} \mathrm{C}$ for $X c T D O$ ). Cells were harvested by centrifugation at $4,000 \mathrm{~g}$ for 15 minutes at $4{ }^{\circ} \mathrm{C}$ and pellets stored at $-80^{\circ} \mathrm{C}$.

Purification of TDO. Cell pellets of harvested cells as above were re-suspended in $50 \mathrm{ml}$ lysis buffer (50 mM NaH $2 \mathrm{PO}_{4}, 300 \mathrm{mM} \mathrm{NaCl}, 10 \mathrm{mM}$ imidazole, $\mathrm{pH}$ 8.0) supplemented with two cOmplete protease inhibitor tablets (Roche) and lysed by the addition of lysozyme $(5 \mathrm{mg}$ ) and by sonication (on ice, 6 × 30 second pulses with 1 minute intervals using an MSE Soniprep 150 sonicator). After sonication, DNase I (5 mg) and $\mathrm{MgCl}_{2}$ (to a final concentration of $20 \mathrm{mM}$ ) were added and the suspension stirred for 30 minutes at $4{ }^{\circ} \mathrm{C}$. The lysate was then centrifuged at $38,000 \mathrm{~g}$ for 50 minutes. A $20 \mathrm{ml}$ column of Ni-NTA superflow resin (Qiagen) was equilibrated with lysis buffer and the cell free extract loaded onto the column. The resin was washed with 15 column volumes of wash buffer (50 mM NaH $2 \mathrm{PO}_{4}, 300 \mathrm{mM} \mathrm{NaCl}, 20 \mathrm{mM}$ imidazole, $\mathrm{pH}$ 8.0) and the protein eluted using a linear gradient of $20-250 \mathrm{mM}$ imidazole in $50 \mathrm{mM} \mathrm{NaH} \mathrm{PO}_{4}, 300 \mathrm{mM} \mathrm{NaCl} \mathrm{pH} 8.0$ buffer. A pre-packed column (HiLoad 16/60 Superdex 200, GE Healthcare) was equilibrated with $50 \mathrm{mM}$ Tris, $100 \mathrm{mM}$ $\mathrm{NaCl}, \mathrm{pH} 8.0$ using an AKTA FPLC (GE Healthcare). Protein samples $(2 \mathrm{ml})$ were injected onto the column and subsequently eluted using a flow rate of $1 \mathrm{ml} /$ minute collected as $1 \mathrm{ml}$ fractions (absorbance measured at $280 \mathrm{~nm}$ ). Fractions were analysed using SDS-PAGE and TDO-containing fractions were pooled and dialysed against $50 \mathrm{mM}$ Tris, $\mathrm{pH}$ 8.0. Samples of hTDO and XcTDO were 
reconstituted with hemin (Sigma) prior to storage at $-80^{\circ} \mathrm{C}$. Hemin $(1.5$ equivalents, made up in 10 $\mathrm{mM} \mathrm{NaOH}$ ) was added to the protein stock and then incubated on ice in the dark for $60-120$ minutes. Free hemin was removed by passage of the protein through a small gel filtration column (Bio-Rad 10 DG) before being concentrated using a Millipore Amicon Ultra $30 \mathrm{kDa}$ filtration unit. Protein concentrations were determined using reported absorption coefficients (hTDO $\varepsilon_{408}=196 \mathrm{mM}^{-1} \mathrm{~cm}^{-1} 16$ and $X c$ TDO $\varepsilon_{404}=180.5 \mathrm{mM}^{-1} \mathrm{~cm}^{-18}$ ).

Anaerobic sample handling. Anaerobic buffers were prepared by bubbling with $\mathrm{O}_{2}$-free nitrogen gas for $\sim 60-120$ minutes. Buffers were then stored in an anaerobic glove box ((Belle Technology Ltd, $\left[\mathrm{O}_{2}\right]$ $<5 \mathrm{ppm}$ ) overnight to remove residual traces of oxygen. Solutions of sodium dithionite and enzyme substrates were prepared by adding the appropriate solid to anaerobic buffer inside the glove box. For preparation of buffer at differing concentrations of oxygen, buffers $(\sim 5 \mathrm{ml})$ were saturated with $\mathrm{O}_{2}$ by bubbling with oxygen gas for $\sim 15$ minutes in a septum sealed flask. Final $\mathrm{O}_{2}$ concentrations were calculated on the basis of a saturating concentration of $\mathrm{O}_{2}$ in solution of $\sim 1.2 \mathrm{mM}$ at $25{ }^{\circ} \mathrm{C}$ and $1 \mathrm{~atm}$. The ferrous forms of hTDO and XcTDO were generated by addition of microlitre volumes (1-5 $\mu \mathrm{l})$ of sodium dithionite (prepared in anaerobic solutions of $50 \mathrm{mM}$ Tris- $\mathrm{HCl}$ buffer, $\mathrm{pH} 8.0$ ) to the ferric enzyme. Reduction was monitored spectrophotometrically using a JASCO V630 spectrophotometer housed in a Belle Technology glove box until no further spectral changes occurred. Equilibrium binding constants $\left(K_{\mathrm{D}}\right)$ were determined by addition of microlitre volumes $(0.5-2.0 \mu \mathrm{l})$ of ligand from an appropriate stock solution (made up in $50 \mathrm{mM}$ Tris- $\mathrm{HCl}$ buffer, $\mathrm{pH}$ 8.0) to ferric hTDO or XcTDO $(1-5 \mu \mathrm{M})$ until no further spectral changes occurred. Ligand binding to ferrous $X c \mathrm{TDO}$ (prepared by stoichiometric titration of ferric $X c \mathrm{TDO}$ with sodium dithionite prior to the titration) was carried out anaerobically and all binding constants were calculated as previously described ${ }^{16}$.

Steady state kinetics. Enzyme activities were measured by monitoring the formation of NFK at 321 $\mathrm{nm}$. Reactions were performed at $25{ }^{\circ} \mathrm{C}$ in $50 \mathrm{mM}$ Tris- $\mathrm{HCl}$ buffer, $\mathrm{pH} 8.0$ containing: $10 \mu \mathrm{M}$ methylene blue, $100 \mu \mathrm{g}$ of catalase, $20 \mathrm{mM}$ L-ascorbate and a fixed concentration of enzyme (typically 100-300 nM). The reaction was initiated by the addition of substrate and initial rates calculated from the absorbance increase at $321 \mathrm{~nm}\left(\varepsilon_{321}=3750 \mathrm{M}^{-1} \mathrm{~cm}^{-1}\right)$. Kinetic parameters $\left(k_{\mathrm{cat}}\right.$ and $K_{\mathrm{M}}$ values $)$ were determined by fitting the data to the Michaelis-Menten equation. 
Pre-steady state kinetics. Stopped flow experiments were carried out using an Applied Photophysics SX.20MV stopped flow spectrometer housed in an anaerobic glove box (Belle Technology Ltd., $\left[\mathrm{O}_{2}\right]$ $<5 \mathrm{ppm})$ and fitted with a Neslab RTE-200 circulating water bath $\left(25.0 \pm 0.1{ }^{\circ} \mathrm{C}\right)$. Multiplewavelength absorption studies were carried out using a photodiode array detector and Pro SX software (Applied Photophysics Ltd.). Spectral deconvolution was performed by global analysis and numerical integration methods using PROKIN software (Applied Photophysics Ltd.). Kinetic data were fitted to an appropriate model (e.g. $\mathrm{A} \rightarrow \mathrm{B} \rightarrow \mathrm{C}$ ). Model validity was assessed using the following criteria: lack of systematic deviations of the residual plot; inspection of calculated spectra to ensure they are consistent with heme spectra; and confirmation that the number of significant singular values following singular value decomposition is consistent with the fitted model. The stated XcTDO, hTDO and reagent concentrations relate to final concentrations after mixing. Single mixing stopped flow experiments were initiated by mixing ferrous TDO $(2-5 \mu \mathrm{M})$ with substrate(s) (e.g. $\mathrm{O}_{2}$, hydrogen peroxide and tryptophan) and the resulting spectral changes were monitored. Formation of NFK was followed at $321 \mathrm{~nm}$ and the decay of an unknown intermediate at $577 \mathrm{~nm}$. In all cases substrate was present in excess compared with the $\mathrm{O}_{2}$ concentration and decay of any intermediates led to formation of ferrous heme at the end of the experiment. For sequential mix experiments using peroxide to form Compound II, ferrous hTDO $(4.5 \mu \mathrm{M})$ was firstly mixed with 10 equivalents of $\mathrm{H}_{2} \mathrm{O}_{2}$ and the solution aged for $50 \mathrm{~ms}$ (to ensure complete formation of Compound II) before a second mix with either buffer or L-Trp (1 mM); the resulting spectral changes were monitored.

Mass spectrometry. Samples were prepared in an anaerobic glove box. Ferrous XcTDO or hTDO (0.5 $-1 \mu \mathrm{M}$, generated by stoichiometric titration of ferric hTDO or XcTDO with sodium dithionite) was incubated with substrate ([substrate] $\geq 10 \times K_{\mathrm{M}}$ in cases where $K_{\mathrm{M}}$ is known) prior to the addition of aerobic solutions of buffer $\left(\left[\mathrm{O}_{2}\right]=258 \mu \mathrm{M}, 50 \mathrm{mM}\right.$ Tris- $\mathrm{HCl}$ buffer, $\mathrm{pH}$ 8.0). Mass spectrometry samples were also prepared using the steady state assay (see above). Samples were allowed to react for varying amounts of time (10 - 60 minutes) before being centrifuged (13,000 rpm, 3 minutes) and the supernatant frozen directly on dry ice as previously described ${ }^{19}$. Samples were stored at $-80{ }^{\circ} \mathrm{C}$ until required for mass spectrometry analysis. Samples were analysed by LC-MS using a Xevo QTof mass spectrometer (Waters) coupled to an Acquity LC system (Waters) using an Acquity UPLC BEH C18 column $(2.1 \times 50 \mathrm{~mm}$, Waters $)$. A linear gradient $(95 \%-0 \%$ solvent A $(0.1 \%$ formic acid in water $)$ and 
5\%-100\% solvent B $(0.1 \%$ formic acid in acetonitrile $))$ was run over 3 minutes at a flow rate of $0.6 \mathrm{ml}$ per minute. The ESI capillary voltage was $3 \mathrm{kV}$, cone voltage $30 \mathrm{~V}$ and collision energy $4 \mathrm{eV}$. The acquisition rate was 10 spectra per second and $\mathrm{m} / \mathrm{z}$ data ranging from 50 to $2000 \mathrm{Da}$ were collected. Mass accuracy was achieved using a reference lock mass scan, once every 10 seconds.

\section{RESULTS}

The identification of reaction intermediates formed during TDO turnover is not straightforward: it depends on being able to differentiate the initially-formed ferrous-oxy species from other intermediates that accumulate during turnover. We designed anaerobic stopped-flow experiments to identify the various catalytic species formed during the $\mathrm{O}_{2}$-dependent reaction of TDO with L-Trp.

Formation of ferrous-oxy TDO. While formation of ferrous-oxy IDO is established, see for example ${ }^{11,20,21}$, there is as yet no evidence for a discrete ferrous-oxy species in the absence of substrate in any $\mathrm{TDO}^{8,22}$.

For hTDO under conditions of high concentrations of oxygen $(c a .600 \mu \mathrm{M})$, we successfully identified a species $\left(\lambda_{\max }=414,544,576 \mathrm{~nm}\right.$, Figure $1 \mathrm{~A}$ (green spectrum)) from the global analysis data. This spectrum is consistent with ferrous-oxy formation and the maxima are similar to those for the same ferrous-oxy species in IDO $\left(\lambda_{\max }=416,543\right.$, and $\left.577 \mathrm{~nm}\right)$. This ferrous-oxy intermediate is relatively unstable and decays to ferric with a first order rate constant $k_{\mathrm{obs}} \approx 4 \mathrm{~s}^{-1}$, Figure 1A. Similar wavelength maxima were identified for reaction of hTDO at lower concentrations of $\mathrm{O}_{2}(c a .130 \mu \mathrm{M})$, but in this case incomplete formation of the ferrous-oxy species occurs (data not shown).

No such ferrous-oxy species (as evidence by the expected peaks at $\approx 540$ and $\approx 580 \mathrm{~nm}$ ) could be observed in an identical set of experiments on $X c \mathrm{TDO}$ - in this case the ferrous enzyme was observed to decay slowly $\left(k_{\mathrm{obs}} \approx 2.5 \mathrm{~s}^{-1}\right.$ ) back to ferric without an observable oxy species (with maxima at $\approx 544$ and $\approx 576 \mathrm{~nm}$ ) in between, Figure S1. We do not have an explanation for fact that hTDO can form a ferrous oxy species in the absence of Trp but XcTDO cannot.

Detection of intermediates during turnover. We then examined oxidation of L-Trp by hTDO and $X c$ TDO, to identify the intermediates formed under turnover conditions. 
In a single mixing anaerobic stopped flow experiment, ferrous hTDO or ferrous XcTDO were mixed with oxygen and L-Trp and monitored over long time scales (10-100 s). The first species (collected at $20 \mathrm{~ms}$ after mixing) showed spectral characteristics $\left(\lambda_{\max }=417,544,577 \mathrm{~nm}\right.$ for hTDO and $\lambda_{\max }=$ $418,548,576 \mathrm{~nm}$ for $X c$ TDO) similar to those for the ferrous-oxy complex above. This species is formed immediately and was assigned as a ternary [ $\mathrm{Fe}(\mathrm{II})-\mathrm{O}_{2}$, L-Trp] complex on the basis of the following observations. (i) The spectrum of the ternary complex formed for XcTDO in the presence of L-Trp (Figure 1C, below) has sharp features in the visible region and is distinct from the behaviour in the absence of L-Trp when no such features are observed (as above). (ii) Exactly the same ternary species was observed in the pre-steady state $\left(\lambda_{\max }=417,544,577 \mathrm{~nm}\right.$ for hTDO and $\lambda_{\max }=417,548$, $576 \mathrm{~nm}$ for $X c \mathrm{TDO}$, Figure S2A, B (spectra shown in red)) in a separate single mixing stopped flow experiment where ferrous enzyme was mixed with both substrates and allowed to react for only 100 ms (i.e. sufficient time to allow formation of a ternary complex but not long enough to allow for NFK production). (iii) The shape of the spectra and the wavelength maxima for the ternary complexes of hTDO and XcTDO are similar to one another and are in very good agreement with those previously observed $^{11}$ for the ternary $\left[\mathrm{Fe}(\mathrm{II})-\mathrm{O}_{2}, \mathrm{~L}-\mathrm{Trp}\right]$ complex of hIDO. A comparison of spectra for all of these ternary complexes (formed within a few ms of mixing) is in Figure 1D, and demonstrates the similarity.

Following initial formation of the ternary complex (within the first few ms), there is a lag phase of $\approx$ $800 \mathrm{~ms}$ for hTDO and $\approx 400 \mathrm{~ms}$ for XcTDO; at the end of the lag phase, NFK formation begins (as observed by an increase in absorbance at $321 \mathrm{~nm}$ ) and the system reaches steady state. Over these longer timescales the ternary complex as observed above persists and with essentially the same spectra as observed above; Figures $1 \mathrm{~B}$ and $1 \mathrm{C}$ (red spectra) show the spectrum of this ternary complex just prior to NFK formation (i.e. at the end of the lag phase). After this point, the ternary complex decays and there is a direct correlation between NFK formation (observed at $321 \mathrm{~nm}$ ) and decay of the ternary complex (monitored at $577 \mathrm{~nm}$, Figure 1B, C insets) as oxygen is consumed to form the product.

Reactivity with other substrates. Unlike IDO which has wide substrate specificity, the range of substrates that are oxidised by TDO is unimpressive. Hence, it is not feasible to examine the formation of reaction intermediates during the oxidation of a variety of other substrates (as was the case in $\left.\mathrm{IDO}^{11}\right)$. 
However, we were able to observe TDO activity with 5-fluoro-Trp (5-F-Trp, Scheme 1). In a parallel set of experiments to those described for L-Trp above, the pre-steady state reactivity of 5-F-Trp by XcTDO was also examined. The stopped-flow data for 5-F-Trp were found to be similar to those for oxidation of L-Trp, Figure S3, and a ternary complex is identified $\left(\lambda_{\max }=417,546,576 \mathrm{~nm}\right.$ Figure S3 (red spectrum)) and accumulates prior to NFK formation. The decay of this ternary complex also correlates with product formation (Figure S3, inset); separate mass spectrometry analyses confirmed the formation of 5-F-NFK as product ( $\mathrm{m} / \mathrm{z}=255$, data not shown).

We also observed binding $\left(K_{\mathrm{D}, \mathrm{IPA}}=341 \pm 37 \mu \mathrm{M}\right)$ of IPA, Scheme 1, to ferrous $X c \mathrm{TDO}$ and with both $X c$ TDO and hTDO we identified a ternary $\left[\mathrm{Fe}(\mathrm{II})-\mathrm{O}_{2}\right.$, IPA $]$ complex (data not shown) and formation of product (IPA-NFK, $\mathrm{m} / \mathrm{z}=222$ ). The overall kinetic profile with IPA is similar to that reported for $\mathrm{hIDO}^{11}$ and the data provide the first evidence for the oxidation of IPA by TDO.

Independent formation of Compound II in TDO. A Compound II species, as observed in IDO during turnover ${ }^{l l}$, is not identified in any of the kinetic transients above. We independently verified the capability of TDO for Compound II (ferryl) formation, by reaction of ferrous TDO with $\mathrm{H}_{2} \mathrm{O}_{2}$ (which leads directly to Compound II). The spectra of Compound II thus formed $\left(\lambda_{\max }=418,529,558 \mathrm{~nm}\right.$ for hTDO; $\lambda_{\max }=411,528,552 \mathrm{~nm}$ for $X c \mathrm{TDO}$ ) are shown in Figure 2A, B. These spectra for TDO are clearly different from the spectra of the ternary complexes that accumulate during the steady state as above, as indicated in Figure 2C.

We also obtained the spectrum of Compound II for hTDO in the presence of L-Trp (by direct reaction of Compound II, formed as above, with L-Trp, Figure S4). The spectrum of this species $\left(\lambda_{\max }=419\right.$, $556,586^{\text {sh }} \mathrm{nm}$, Figure S4B) is different from that of TDO Compound II without substrate present (compare Figures 2A and S4A) and we assign this new species as a [Compound II, L-Trp] complex. Note that the spectrum of this (artificially formed) [Compound II, L-Trp] complex in TDO (Figure S4B) is also not the same as that of the same IDO [Compound II, L-Trp] complex formed in the steady state (or, of Compound II of IDO formed from Fe(II) and $\mathrm{H}_{2} \mathrm{O}_{2}$ ), as the IDO species contains a distinct and characteristic shoulder at $593 \mathrm{~nm}^{11}$ (Figure 1E). The reportedly higher affinity of L-Trp for IDO than for TDO might account for this difference in the spectra.

Looking at the kinetic data as a whole, the most important points are: (i) TDO is, like most heme proteins, capable of Compound II formation (others ${ }^{23}$ have drawn similar conclusions about the 
intrinsic ability of TDO to form a ferryl heme), Figure 2. (ii) Neither of the Compound II species formed above for TDO by reaction with $\mathrm{H}_{2} \mathrm{O}_{2}$ (in the absence, Figure 2, and presence, Figure S4, of substrate) can be observed in the steady state, Figure 2C. (iii) The species that accumulate in the steady state for IDO and TDO are not the same (Figure 1E).

\section{DISCUSSION}

The IDO and TDO enzymes are very similar in their function and properties - they were discovered at about the same time, and in fact were often confused with one another in the early literature. This has left an invisible imprint on the literature, so that IDO and TDO are often considered to be two halves of a matching pair. Perhaps because of this, IDO and TDO have been assumed (including by us) to react by a similar mechanism. But actually there are some persistent differences between the two enzymes - for example the much wider substrate specificity in IDO than in TDO, the relative instability of the ferrous-oxy species in TDO in the absence of substrate, and the (still largely unexplained) observation ${ }^{16,24}$ that ferric TDO is, uniquely, also able to oxidise tryptophan without any apparent need for a reducing substrate. The possibility has always therefore existed that they might not react by exactly the same mechanism; and, what is more, there is no evidence that they do. The source of greatest anxiety to these authors has been the fact that the species corresponding to Compound II in IDO is notable by its absence in TDO.

In IDO, the formation of a Compound II species during turnover has been implicated on the basis of resonance Raman ${ }^{9,10}$ and uv-visible ${ }^{11}$ spectra, and is supported by computational analyses ${ }^{12,13,15}$. That Compound II is detected at all must mean that its decay is rate-limiting in the steady state. The kinetic techniques that have allowed us to extract convincing spectra for Compound II species under steady state (turnover) conditions in IDO $^{l l}$ have, in this work, yielded no such evidence for a Compound II species in either hTDO or $X c$ TDO. But the kinetic experiments do reveal new information on the TDO mechanism and they allow helpful comparisons with IDO, as explained below and summarized in Scheme 2.

(i) The ferrous-oxy form of TDO. Considering that TDO is an $\mathrm{O}_{2}$-dependent heme enzyme, it has proved much more difficult than one would have expected to isolate a ferrous-oxy 
intermediate in any TDO in the absence of substrate. Using somewhat forcing (i.e. high, probably non-physiological) concentrations of $\mathrm{O}_{2}$, we can detect a ferrous-oxy species in hTDO (Figure 1A), which by comparison with $\operatorname{IDO}^{11,20}$ we believe to be convincing. It demonstrates the feasibility of ferrous-oxy formation in TDO, which is consistent with the mechanism shown in Scheme 2 below, but probably means that L-Trp binds first in the mechanism (as the ternary [Fe(II)- $\mathrm{O}_{2}, \mathrm{~L}-\mathrm{Tr}$ ] complex is formed more easily).

(ii) Pre-steady state - the ternary [Fe(II)- $\mathrm{O}_{2}, \mathrm{~L}$-Trp] complex. In the pre-steady state, we have detected formation of a ternary [Fe(II)- $\mathrm{O}_{2}$, L-Trp] complex. This species forms rapidly (within a few milliseconds) in both hTDO and $X c$ TDO, and the formation of this complex and its spectrum are not affected by the order in which the substrates $\left(\mathrm{O}_{2} / \mathrm{Trp}\right)$ are added (sequentially, or together). The behaviour is similar to that for IDO in which a ternary complex is also reported ${ }^{11}$, and the shape of the spectra and their wavelength maxima for IDO and TDO (Figure 1D, Table 1) are in good agreement. .

(iii) Steady state. In both TDOs that we examined, formation of the initial [Fe(II)- $\left.\mathrm{O}_{2}, \mathrm{~L}-\mathrm{Trp}\right]$ ternary complex is followed by a lag phase which precedes product (NFK) formation. During the lag phase, the ternary complex persists. It is this ternary species that accumulates during TDO turnover, and on the same time scales $(0.5-1 \mathrm{~s})$ as the accumulation of Compound II during IDO turnover ${ }^{11}$. After the lag phase is over (about 1 s after mixing), decay of the ternary complex (at $577 \mathrm{~nm}$ ) occurs in parallel with the formation of NFK (at $321 \mathrm{~nm}$ ), which connects the ternary complex to the catalytic formation of product.

The steady state behaviour of the TDOs is notably different from IDO. In IDO, a Compound II species is identified in the steady state and is distinct from the initiallyformed ternary complex ${ }^{11}$. For TDO, no such Compound II is identified in our experiments. But the absence of Compound II in the steady state in TDO is not down to an intrinsic inability of the TDO enzyme to form ferryl heme, because Compound II formation is possible through a different route in which ferrous heme is reacted with peroxide, Figure 2 (IDO is similarly competent for ferryl heme formation with peroxide $\left.^{25}\right)$. 
(iv) Steady state - comparison of spectroscopic properties of the ternary complex in TDO and Compound II in IDO. Wavelength maxima for all of the relevant species are summarised in Table 1, for direct comparison. A comparison of the spectra identified under steady state conditions for IDO and TDO is presented in Figure 1E, and demonstrates the difference in the spectroscopic properties. Notably, the Compound II intermediate of IDO has a definite $593 \mathrm{~nm}$ shoulder, but the TDO intermediate does not. The Q bands in the TDO ternary complex are sharper, more reminiscent of a genuine ferrous-oxy spectrum (such as that in myoglobin for example ${ }^{26}$, or in $\mathrm{IDO}^{20}$ ), and there is little absorption in the $>600 \mathrm{~nm}$ region; the Q bands in IDO Compound II are "squashed" by comparison, with a trailing absorbance still observable at wavelengths $>650 \mathrm{~nm}$.

\begin{abstract}
All of the data suggest that the ternary $\left[\mathrm{Fe}(\mathrm{II})-\mathrm{O}_{2}, \mathrm{~L}-\mathrm{Trp}\right]$ complex and not Compound II, accumulates during turnover of TDO. So why is there a difference between IDO and TDO? The simplest interpretation is that the IDO and TDO enzymes use the same mechanism, but that the rate-limiting step is not the same. Scheme 2 gives a pictorial summary of our ideas. We propose that in IDO, decay of Compound II is rate-limiting and thus Compound II accumulates. In TDO, there is a change in the rate-limiting step such that Compound II never accumulates; instead, the ternary complex is observed in the steady state and its decay is rate-limiting. An alternative possibility that is worth considering to account for the difference is that TDO uses an electrophilic addition route, Scheme 2, whereas IDO uses radical addition. That the ferrous oxy species of IDO is thought to contain superoxide character ${ }^{9}$, and that there is evidence in $\mathrm{TDO}^{22}$ that electrophilic addition is favoured over radical addition, would be consistent with this explanation. As noted recently $y^{22}$, further work is necessary to fully understand the mechanism of tryptophan oxidation.
\end{abstract}


1

2

3

4

5

6

7

8

9

\section{AUTHOR INFORMATION.}

Corresponding author. Department of Chemistry, University of Leicester, University Road, Leicester, LE1 7RH, England UK. Telephone: +44 (0)116 2297047; Fax +44 (0)116 252 2789; Email: emma.raven@le.ac.uk.

Funding. This work was supported by grants from EPSRC (studentship to EB) and BBSRC (BB/L004585/1, fellowship to ER).

Author contributions. ER and SH conceived and coordinated the work. JB and EB collected the kinetic data. JB, EB and ER analysed the kinetic data. ML collected and analyzed the mass spectrometry data. ER coordinated writing of the paper, with assistance from all authors.

\section{ASSOCIATED CONTENT.}

Supporting information. The Supporting Information is available free of charge on the ACS Publications website at www.xxx.xxx.xxx. Reactivity of $X c \mathrm{TDO}$ with $\mathrm{O}_{2}$ (Figure S1), formation of the ternary [Fe(II)- $\mathrm{O}_{2}$, L-Trp] complex in TDOs (Figure S2), oxidation of 5-fluoro-Trp by ferrous $X c$ TDO (Figure S3) and formation of Compound II in TDO in the presence of substrate (Figure S4). 


\section{REFERENCES}

[1] Hayaishi, O., Rothberg, S., Mehler, A. H., and Saito, Y. (1957) Studies on oxygenases; enzymatic formation of kynurenine from tryptophan, J Biol Chem 229, 889-896.

[2] Millett, E. S., Efimov, I., Basran, J., Handa, S., Mowat, C. G., and Raven, E. L. (2012) Hemecontaining dioxygenases involved in tryptophan oxidation, Curr Opin Chem Biol 16, 60-66.

[3] Geng, J., and Liu, A. (2014) Heme-dependent dioxygenases in tryptophan oxidation, Arch Biochem Biophys 544, 18-26.

[4] Hamilton, G. A. (1969) Mechanisms of two- and four-electron oxidations catalyzed by some metalloenzymes, Adv. Enzymol. Relat. Areas Mol. Biol. 32, 55-96.

[5] Chauhan, N., Thackray, S. J., Rafice, S. A., Eaton, G., Lee, M., Efimov, I., Basran, J., Jenkins, P. R., Mowat, C. G., Chapman, S. K., and Raven, E. L. (2009) Reassessment of the Reaction Mechanism in the Heme Dioxygenases, $J$ Am Chem Soc 131, 4186-+.

[6] Geng, J., Dornevil, K., and Liu, A. (2012) Chemical rescue of the distal histidine mutants of tryptophan 2,3-dioxygenase, J Am Chem Soc 134, 12209-12218.

[7] Lu, C., Lin, Y., and Yeh, S. R. (2009) Inhibitory substrate binding site of human indoleamine 2,3dioxygenase, J Am Chem Soc 131, 12866-12867.

[8] Thackray, S. J., Bruckmann, C., Anderson, J. L., Campbell, L. P., Xiao, R., Zhao, L., Mowat, C. G., Forouhar, F., Tong, L., and Chapman, S. K. (2008) Histidine 55 of tryptophan 2,3dioxygenase is not an active site base but regulates catalysis by controlling substrate binding, Biochemistry 47, 10677-10684.

[9] Lewis-Ballester, A., Batabyal, D., Egawa, T., Lu, C., Lin, Y., Marti, M. A., Capece, L., Estrin, D. A., and Yeh, S. R. (2009) Evidence for a ferryl intermediate in a heme-based dioxygenase, Proc Natl Acad Sci U S A 106, 17371-17376.

[10] Yanagisawa, S., Yotsuya, K., Hashiwaki, Y., Horitani, M., Sugimoto, H., Shiro, Y., Appelman, E. H., and Ogura, T. (2010) Identification of the $\mathrm{Fe}-\mathrm{O} 2$ and the $\mathrm{Fe}=\mathrm{O}$ heme species for indoleamine 2,3-dioxygenase during catalytic turnover, Chem. Lett. 39, 36-37.

[11] Booth, E. S., Basran, J., Lee, M., Handa, S., and Raven, E. L. (2015) Substrate Oxidation by Indoleamine 2,3-Dioxygenase J Biol Chem 290, 30924-30930. 
[12] Chung, L. W., Li, X., Sugimoto, H., Shiro, Y., and Morokuma, K. (2008) Density functional theory study on a missing piece in understanding of heme chemistry: the reaction mechanism for indoleamine 2,3-dioxygenase and tryptophan 2,3-dioxygenase, $J$ Am Chem Soc 130, 12299-12309.

[13] Chung, L. W., Li, X., Sugimoto, H., Shiro, Y., and Morokuma, K. (2010) ONIOM study on a missing piece in our understanding of heme chemistry: bacterial tryptophan 2,3-dioxygenase with dual oxidants, J Am Chem Soc 132, 11993-12005.

[14] Capece, L., Lewis-Ballester, A., Yeh, S. R., Estrin, D. A., and Marti, M. A. (2012) Complete reaction mechanism of indoleamine 2,3-dioxygenase as revealed by QM/MM simulations, $J$ Phys Chem B 116, 1401-1413.

[15] Capece, L., Lewis-Ballester, A., Batabyal, D., Di Russo, N., Yeh, S. R., Estrin, D. A., and Marti, M. A. (2010) The first step of the dioxygenation reaction carried out by tryptophan dioxygenase and indoleamine 2,3-dioxygenase as revealed by quantum mechanical/molecular mechanical studies, J Biol Inorg Chem 15, 811-823.

[16] Basran, J., Rafice, S. A., Chauhan, N., Efimov, I., Cheesman, M. R., Ghamsari, L., and Raven, E. L. (2008) A kinetic, spectroscopic, and redox study of human tryptophan 2,3-dioxygenase, Biochemistry 47, 4752-4760.

[17] Forouhar, F., Anderson, J. L., Mowat, C. G., Vorobiev, S. M., Hussain, A., Abashidze, M., Bruckmann, C., Thackray, S. J., Seetharaman, J., Tucker, T., Xiao, R., Ma, L. C., Zhao, L., Acton, T. B., Montelione, G. T., Chapman, S. K., and Tong, L. (2007) Molecular insights into substrate recognition and catalysis by tryptophan 2,3-dioxygenase, Proc Natl Acad Sci U S A $104,473-478$.

[18] Chauhan, N., Basran, J., Rafice, S. A., Efimov, I., Millett, E. S., Mowat, C. G., Moody, P. C. E., Handa, S., and Raven, E. L. (2012) How is the distal pocket of a heme protein optimized for binding of tryptophan?, Febs $J$ 279, 4501-4509.

[19] Basran, J., Efimov, I., Chauhan, N., Thackray, S. J., Krupa, J. L., Eaton, G., Griffith, G. A., Mowat, C. G., Handa, S., and Raven, E. L. (2011) The mechanism of formation of Nformylkynurenine by heme dioxygenases, J Am Chem Soc 133, 16251-16257. 
[20] Rosell, F. I., Kuo, H. H., and Mauk, A. G. (2011) NADH oxidase activity of indoleamine 2,3dioxygenase, J Biol Chem 286, 29273-29283.

[21] Chauhan, N., Basran, J., Efimov, I., Svistunenko, D. A., Seward, H. E., Moody, P. C., and Raven, E. L. (2008) The role of serine 167 in human indoleamine 2,3-dioxygenase: a comparison with tryptophan 2,3-dioxygenase, Biochemistry 47, 4761-4769.

[22] Makino, R., Obayashi, E., Hori, H., Iizuka, T., Mashima, K., Shiro, Y., and Ishimura, Y. (2015) Initial $\mathrm{O}(2)$ Insertion Step of the Tryptophan Dioxygenase Reaction Proposed by a HemeModification Study, Biochemistry 54, 3604-3616.

[23] Fu, R., Gupta, R., Geng, J., Dornevil, K., Wang, S., Zhang, Y., Hendrich, M. P., and Liu, A. (2011) Enzyme reactivation by hydrogen peroxide in heme-based tryptophan dioxygenase, $J$ Biol Chem 286, 26541-26554.

[24] Batabyal, D., and Yeh, S. R. (2007) Human tryptophan dioxygenase: A comparison to indoleamine 2,3-dioxygenase, J Am Chem Soc 129, 15690-15701.

[25] Lu, C., and Yeh, S. R. (2011) Ferryl derivatives of human indoleamine 2,3-dioxygenase, J Biol Chem 286, 21220-21230.

[26] Antonini, M., and Brunori, E. (1971) Hemoglobin and Myoglobin and their Reactions with Ligands, North Holland Publishers, Amsterdam.

[27] Efimov, I., Basran, J., Thackray, S. J., Handa, S., Mowat, C. G., and Raven, E. L. (2011) Structure and reaction mechanism in the heme dioxygenases, Biochemistry 50, 2717-2724. 
Table 1: Wavelength maxima $(\mathrm{nm})$ for the various species in hTDO and XcTDO.

\begin{tabular}{|c|c|c|}
\hline \multirow[t]{2}{*}{ Derivative } & \multicolumn{2}{|c|}{$\lambda_{\max }$} \\
\hline & hTDO & $X c$ TDO \\
\hline$O x y-\mathrm{Fe}(\mathrm{II})-\mathrm{O}_{2}$ & $414,544,576$ & n.d. \\
\hline Ternary - $\left[\mathrm{Fe}(\mathrm{II})-\mathrm{O}_{2}, \mathrm{~L}-\mathrm{Trp}\right]^{\mathrm{a}}$ & $417,544,577$ & $417,548,576$ \\
\hline Compound $I I^{b}$ & $418,529,558$ & $411,528,552$ \\
\hline
\end{tabular}

n.d. $=$ not detected.

${ }^{\text {a }}$ Accumulates during lag phase of kinetics, prior to steady state turnover (as measured by NFK formation at 321 $\mathrm{nm})$.

${ }^{b}$ Formed by reaction of ferrous enzyme with $\mathrm{H}_{2} \mathrm{O}_{2}$. 


\section{FIGURE LEGENDS}

Scheme 1. Structures of the substrates referred to in this work. (A) L-Trp, (B) 1-Me-L-Trp, (C) 5-F-LTrp, (D) IPA.

Figure 1. (A) Formation of ferrous-oxy hTDO. Stopped-flow spectra showing the formation of ferrous-oxy hTDO. Ferrous hTDO $(2 \mu \mathrm{M})$ was mixed with $\mathrm{O}_{2}(600 \mu \mathrm{M})$ and monitored over $1 \mathrm{~s}$. The black line is the first species observed after mixing and represents the ferrous enzyme (species A below); the green line represents the fully formed ferrous-oxy hTDO complex (species B below) and the dotted line represents the final (oxidised) spectrum (species $\mathrm{C}$ below). Absorbance values in the visible region have been multiplied by a factor of 4 . Inset shows transients monitored at $414 \mathrm{~nm}$ for the reaction shown in the main panel; data were fitted to $\mathrm{A} \rightarrow \mathrm{B} \rightarrow \mathrm{C}$ model, with pseudo-first-order rate constants (obtained from global fitting) of $21.5 \pm 0.4 \mathrm{~s}^{-1}$ (for $\mathrm{A} \rightarrow \mathrm{B}$, corresponding to ferrous-oxy formation) and $3.8 \pm 0.01 \mathrm{~s}^{-1}$ (for B $\rightarrow \mathrm{C}$, corresponding to ferrous-oxy decay). (B), (C) Detection of an intermediate during oxidation of L-Trp by ferrous TDOs. Stopped flow diode-array spectra are shown for (B) hTDO and (C) XcTDO. In each case, ferrous hTDO or XcTDO $(2 \mu \mathrm{M})$ was mixed with $\mathrm{O}_{2}(300 \mu \mathrm{M})$ and L-Trp $(2 \mathrm{mM})$ and monitored over $100 \mathrm{~s}$ (hTDO) or 10s (XcTDO). The red spectrum is that of $\mathrm{hTDO}$ (at $\mathrm{t}=0.8 \mathrm{~s}$ ) and $\mathrm{XcTDO}$ at $\mathrm{t}=0.4 \mathrm{~s}$ ) just prior to NFK formation (at the end of the lag phase), and represents the ternary $\left[\mathrm{Fe}(\mathrm{II})-\mathrm{O}_{2}, \mathrm{~L}-\mathrm{Trp}\right]$ complex. The black line represents the final spectrum. Note that the final spectrum in hTDO is ferric and in XTDO is ferrous. This is because binding of L-Trp to xTDO leads to a large $\left(136 \mathrm{mV}^{8}\right)$ increase in $\mathrm{Fe}^{\mathrm{III}} / \mathrm{Fe}^{\mathrm{II}}$ reduction potential which stabilises the reduced heme formed at the end of the reaction; no increase in reduction potential on binding of substrate is observed for $\mathrm{hTDO}^{16}$, hence the system reverts to ferric at the end of the reaction. Absorbance values in the visible region have been multiplied by a factor of 4 . Insets in (B) and (C) show absorbance changes that report on NFK (at $321 \mathrm{~nm}$ ) and decay of the ternary complex (at $577 \mathrm{~nm}$, absorbance changes multiplied by a factor of 20) over longer timescales. Although $\Delta \mathrm{Abs}$ at $577 \mathrm{~nm}$ is noisy (because of the small absorbancies at this wavelength), the changes are $321 \mathrm{~nm}$ and $577 \mathrm{~nm}$ changes are clearly occurring on the same timescale. (D) Comparison of the spectra of the ternary complexes. Overlay of the spectra for the ternary complex of hIDO (solid line) ${ }^{I 1}$ with those for the ternary complexes obtained in this work for hTDO (dashed line) and XcTDO (dotted line), showing the similarity of the spectra. All spectra are multiplied by a factor of 5 in the visible region, 
for direct comparison; note that the intensity of the $577 \mathrm{~nm}$ peak in the ternary complex for hIDO $(\varepsilon \approx$ $\left.22 \mathrm{mM}^{-1} \mathrm{~cm}^{-1}\right)$ is higher than that for the TDOs $\left(\varepsilon \approx 14 \mathrm{mM}^{-1} \mathrm{~cm}^{-1}\right.$ in both TDOs). (E) Comparison of the intermediates observed during L-Trp oxidation in IDO and TDO. Overlay of the spectrum of Compound II in hIDO (blue line) obtained during turnover with L-Trp ${ }^{11}$, with the spectra of the ternary complex observed under the same conditions during oxidation of L-Trp in hTDO (red dashed line) and XcTDO (red dotted line) taken from Figures 1B and C respectively. Note the absence of the $593 \mathrm{~nm}$ shoulder (observed with hIDO intermediate) in the TDO ternary complex. In all figures, the spectra of the ternary complexes are shown in red and the spectra of Compound II in blue.

Figure 2. Reaction of TDO with $\mathrm{H}_{2} \mathrm{O}_{2}$. (A) Ferrous hTDO $(3 \mu \mathrm{M})$ was mixed with $\mathrm{H}_{2} \mathrm{O}_{2}(10$ equivalents) and monitored over $1 \mathrm{~s}$. Inset shows transients monitored at $430 \mathrm{~nm}$ for the reaction shown in the main panel; data were fitted to $\mathrm{A} \rightarrow \mathrm{B} \rightarrow \mathrm{C}$ model, with pseudo-first order rate constants (obtained from global fitting) of $76.0 \pm 1.5 \mathrm{~s}^{-1}$ (for A $\rightarrow$ B) and $6.1 \pm 0.1 \mathrm{~s}^{-1}$ (for B $\rightarrow$ C). (B) Ferrous XcTDO $(3 \mu \mathrm{M})$ was reacted with $\mathrm{H}_{2} \mathrm{O}_{2}(10$ equivalents) and monitored over $5 \mathrm{~s}$. In each case the dashed line is the first species observed after mixing and represents the ferrous enzyme (species A in the model), the dotted line is a partially oxidised intermediate (species B in the model), and the solid line represents a Compound II (ferryl) species (species C in the model). Absorbance values in the visible region have been multiplied by a factor of 4 (for hTDO) and 5 (XcTDO). Inset shows transients monitored at $432 \mathrm{~nm}$ for the reaction shown in the main panel. The data were fitted to $\mathrm{A} \rightarrow \mathrm{B} \rightarrow \mathrm{C}$ model, with rate constants (obtained from global fitting) of $4.0 \pm 0.01 \mathrm{~s}^{-1}$ (for $\mathrm{A} \rightarrow \mathrm{B}$ ) and $0.2 \pm 0.01 \mathrm{~s}^{-}$ ${ }^{1}$ (for B $\rightarrow$ C). (C) Overlay of the spectra of the ternary [Fe(II)- $\mathrm{O}_{2}$, L-Trp] complex for hTDO (solid red line) and XcTDO (dashed red line) spectra (taken from Figure 1B, C) with the Compound II spectra obtained directly by reaction of ferrous enzyme with $\mathrm{H}_{2} \mathrm{O}_{2}$ for hTDO (solid black line) and $X c$ TDO (dashed black line), showing the difference in the two sets of spectra.

Scheme 2. The mechanism of tryptophan oxidation. The mechanism as drawn above has been the current working model for tryptophan oxidation ${ }^{2,9,27}$. The various intermediates are shown in the same colours as used in Figures 1 and 2 (red: ternary complex; blue; Compound II). In both IDO and TDO, a ternary complex is detected. A change in the rate-limiting step between TDO (black dashed box) and IDO (blue dashed box) accounts for the fact that Compound II does not accumulate in TDO in the 
steady state and the ternary complex (which has a different spectrum to Compound II) accumulates instead.

\section{Scheme 1}

(A)

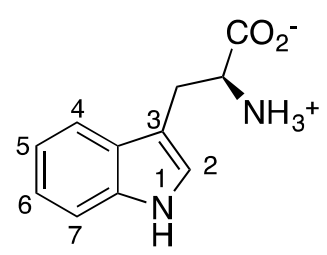

(C)

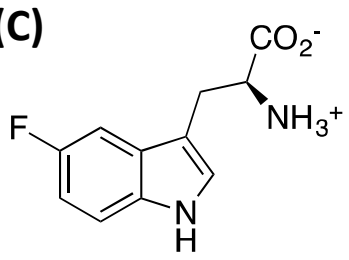

(B)<smiles>Cn1cc(CC([NH3+])C(=O)O)c2ccccc21</smiles>

(D)<smiles>O=C(O)CCc1c[nH]c2ccccc12</smiles> 
(A)

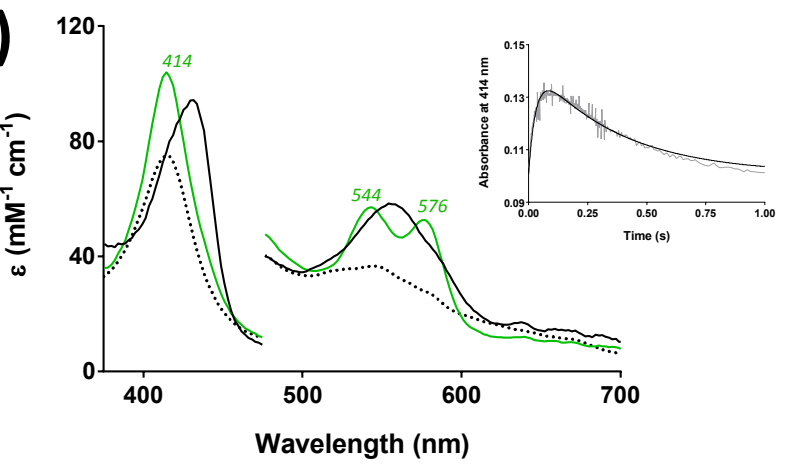

(B)

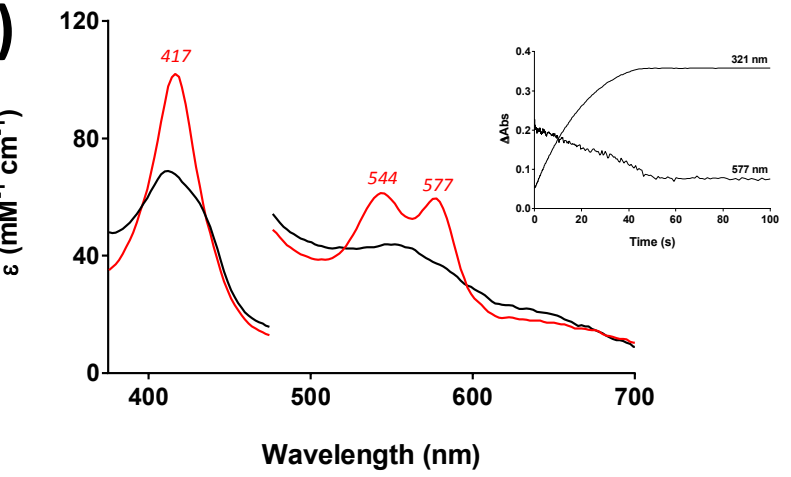

(C)
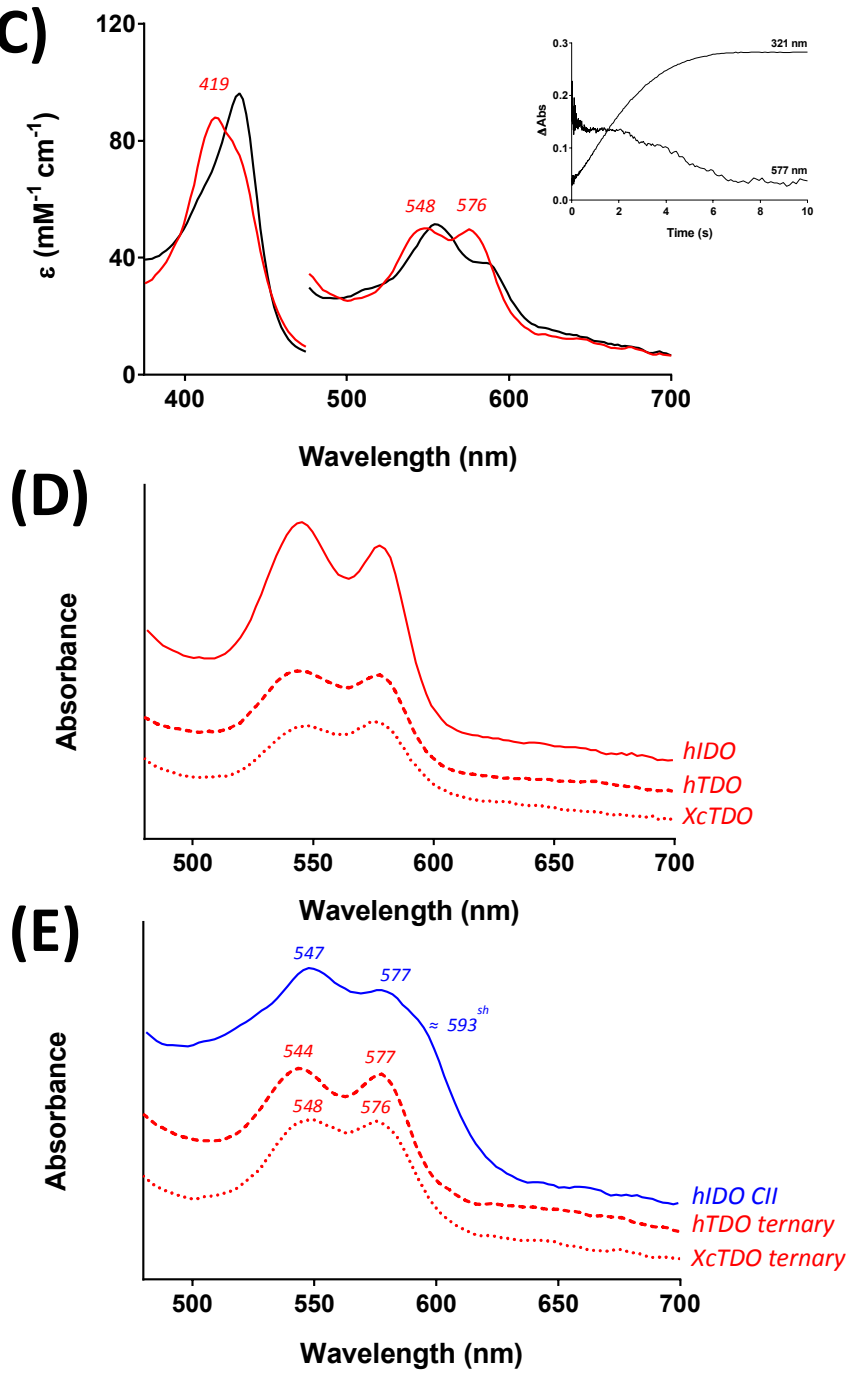

Figure 1 
(A)

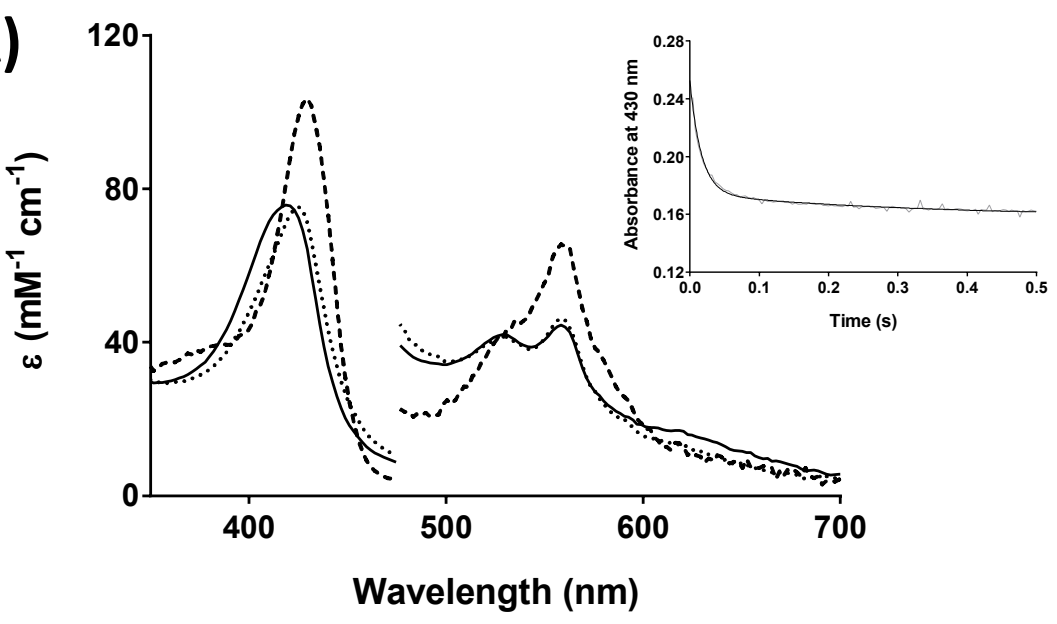

(B)

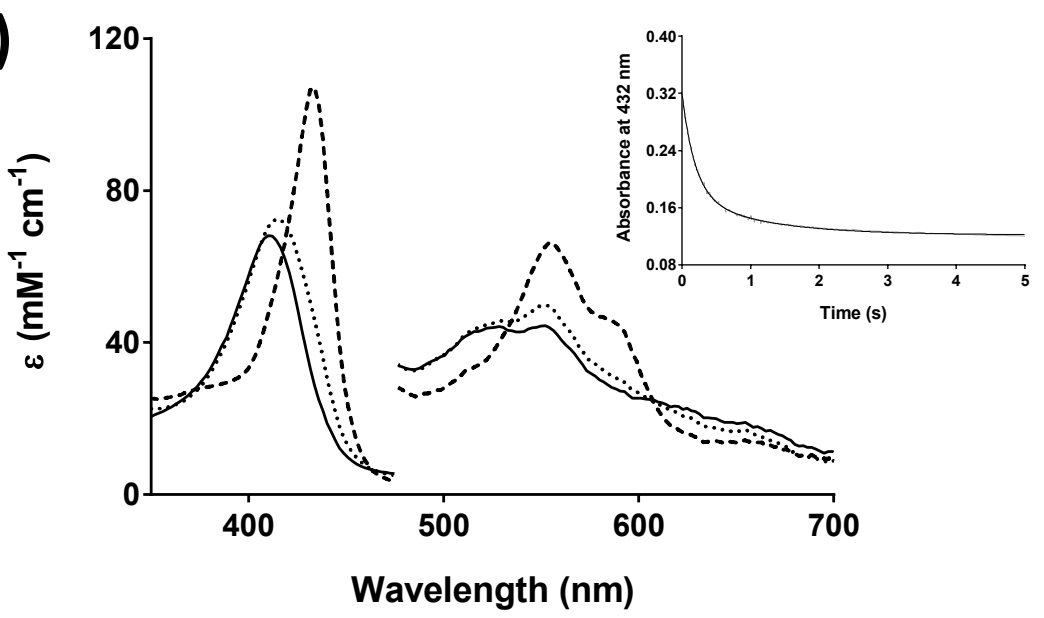

(C)

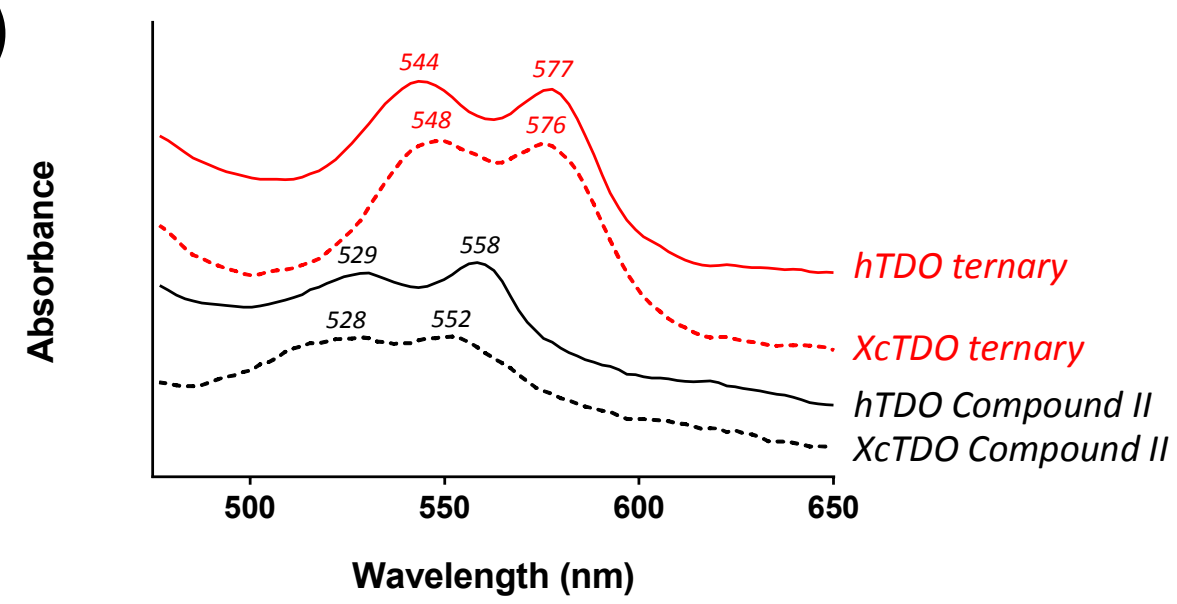



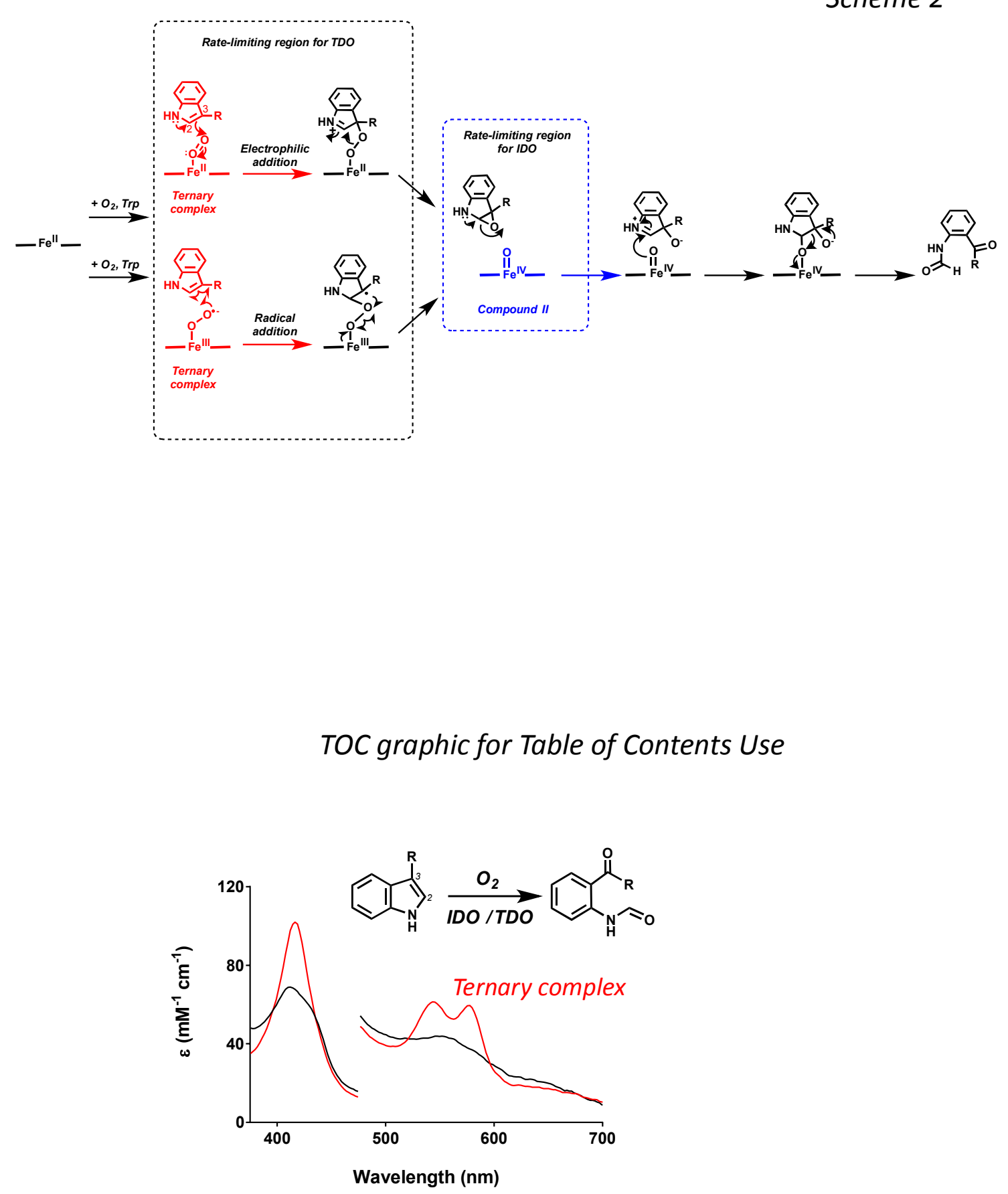